\title{
The cost reduction in hospitalization associated with paliperidone palmitate in the People's Republic of China, Korea, and Malaysia
}

This article was published in the following Dove Press journal:

Neuropsychiatric Disease and Treatment

5 August 2015

Number of times this article has been viewed

\author{
Chiun-Fang Chiou' \\ Bruce CM Wang ${ }^{2}$ \\ Ronald Caldwell ${ }^{3}$ \\ Wesley Furnback ${ }^{2}$ \\ Jung-Sun Lee ${ }^{4}$ \\ Nathan Kothandaraman ${ }^{5}$ \\ SunKyoung Lee ${ }^{6}$ \\ Jin Wang ${ }^{7}$ \\ Fan Zhang ${ }^{8}$
}

'Regional Market Access, Janssen Asia-Pacific, Singapore; 'ㄹlysia Group, LLC, Taipei, Taiwan; ${ }^{3}$ Department of Economics, University of Michigan, Ann Arbor, MI, USA; ${ }^{4}$ Department of Psychiatry, University of Ulsan, College of Medicine, Asan Medical Center, Seoul, Korea; ${ }^{5}$ Government Affairs, Johnson \& Johnson Sdn Bhd, Selangor, Malaysia; ${ }^{6}$ Market Access, Janssen Korea Ltd, Seoul, Korea; ${ }^{7}$ Strategy and Development, Xian Janssen Pharmaceutical Co, Ltd, ${ }^{8}$ Medical Affairs, Xian Janssen Pharmaceutical Co, Ltd, Beijing, People's Republic of China
Correspondence: Bruce CM Wang 333 E 43rd Street, Apartment I0I2, New York, NY 10017 , USA

$\mathrm{Tel}+19176602510$

Fax +l 7328992759

Email bruce.wang@elysiagroup.com
Background: Schizophrenia results in substantial health care utilization costs. Much of these costs can be attributed to health care use resulting from nonadherence to treatment, relapse, and hospitalization.

Aims of the study: The objective of this research is to further estimate the health care resource utilization costs of patients with schizophrenia in the People's Republic of China, Korea, and Malaysia with a specific focus on the reduction in hospitalization costs associated with the use of long-acting, injectable paliperidone palmitate (PP) relative to alternative treatment medications.

Methods: The study focuses exclusively on the estimated reduction in hospitalization days following treatment with PP and the potential associated cost savings. Cost analysis was done using a payer's perspective and only includes direct health care costs associated with hospitalization. Localized cost data were taken from published sources, and health care utilization was estimated based on a clinical study conducted in countries in the Asia-Pacific region. People's Republic of China, Korea, and Malaysia had the highest number of patients enrolled in the clinical study, and thus were chosen for this research. Analysis looked at 12-month and 18-month periods following initial treatment with PP relative to a retrospective 12-month period utilizing alternative treatment medications.

Results: Results suggest that reductions in hospital utilization cost over 12 months may occur through the use of PP relative to alternatives - ranging from \$1,991 for the People's Republic of China to $\$ 6,698$ for Korea and $\$ 6,716$ for Malaysia.

Conclusion: Given the substantial costs associated with the treatment of schizophrenia both worldwide and in Asia, it is important to fully understand the costs and outcomes associated with various treatment options. In this research, we have specifically analyzed the direct health care cost savings associated with hospital utilization for patients taking PP relative to alternative treatment methods. The results suggest that reductions in hospital utilization cost were associated with PP treatment, likely largely due to increased adherence to treatment.

Keywords: schizophrenia, antipsychotic treatment, health economics, cost savings

\section{Introduction}

Schizophrenia is a severe mental disorder that is often characterized by disruptions in thoughts and behaviors that affect the language ability and perceptions of those afflicted with the disease and often cause psychotic experiences resulting in hearing voices or delusions. ${ }^{1}$ While the disease itself varies in severity, sufferers of schizophrenia have an increased risk of suicide with approximately one-third of sufferers attempting suicide at some point in their life and approximately $10 \%$ succeeding in taking their own life. ${ }^{2,3}$ Schizophrenia typically begins early in life, first manifesting in late adolescence 
or early adulthood. ${ }^{1}$ The age for the first episode is typically younger for men, 21 years of age on average, than for women, for whom the average age is 27 years. ${ }^{2,3}$ The exact cause of schizophrenia is unknown, and the possible factors related to onset include chemical imbalances in the brain, genetic factors, or viral infections. ${ }^{4}$

Estimates for the worldwide lifetime prevalence of the disease range from $0.5 \%$ to $1 \%,{ }^{2,3}$ and it is estimated that schizophrenia currently affects 21 million people worldwide. ${ }^{1}$ In the People's Republic of China, schizophrenia is estimated to occur in $0.8 \%$ of the adult population, ${ }^{5}$ and there are $\sim 8$ million currently diagnosed cases of the disease. ${ }^{6}$ The treated prevalence of the disease in Korea in 2005 was estimated to be $\sim 0.4 \%$ of the population. ${ }^{7}$ A 2009 analysis of the Health Insurance Review and Assessment Service-National Patients Sample (HIRA-NPS) in Korea analyzed 126,961 patients with schizophrenia; of these patients, $10.5 \%$ were prescribed with antipsychotic monopharmacy, while $89.5 \%$ were prescribed with polypharmacy. ${ }^{8}$ In Malaysia, the incidence rate of schizophrenia has been reported to be 7.7-43.0 per 100,000 population. ${ }^{9}$

The widespread prevalence of the disease is associated with significant economic burden. An international literature review found that the median health care costs from schizophrenia represented $\sim 1.1 \%$ of total national health care expenditures. ${ }^{10}$ The World Health Organization (WHO) has estimated that the health care costs associated with schizophrenia in the USA and Europe may be as high as $2.6 \%$ of the total health care expenditures. ${ }^{11}$ A US study using a claims database analysis found that patients with schizophrenia had medical care costs four times that of a matched, nonschizophrenic cohort. Of these costs, $\sim 42 \%$ were inpatient expenditures. ${ }^{12}$ A Canadian study estimated the direct health care and nonhealth care costs of schizophrenia to be 2.02 billion Canadian dollars in $2004 .^{13}$ In Korea, the estimated annual, direct health care costs associated with schizophrenia were estimated to be $\$ 418.7$ million in $2005 .{ }^{7}$ A 2009 database analysis of a nationally representative subset of 126,961 patients with schizophrenia in Korea found that the mean total medical cost per patient was US\$3,209. ${ }^{8}$ The direct health care costs per case in the People's Republic of China are estimated to be $\$ 862.81 .^{6}$

Given the extensive health care costs associated with schizophrenia and the amount of these costs that result from hospitalization, it is likely that effective medications could help reduce the incidence of hospitalization and possibly reduce the length of stay in cases where hospitalization is required and therefore potentially result in a reduction in the economic burden associated with the disease.
The primary pharmacological intervention used in the treatment of schizophrenia is antipsychotic drugs. ${ }^{14-16}$ Research suggests that up to $80 \%$ of patients with schizophrenia experience a psychotic relapse within the first 5 years following their initial episode..$^{14,17-19}$ It is thought that treatment nonadherence is a significant factor leading to relapse. ${ }^{14,19}$ In addition to the increased probability of relapse, treatment nonadherence has also been found to be correlated with inconsistent symptom control and hospitalization, potentially leading to a variety of physical and social difficulties for the patient, including progressive loss of brain tissue, possible suicide, family conflict, and loss of independence and employment opportunities. ${ }^{14,20}$ Treatment adherence may be improved through the use of long-acting injectable antipsychotic medications that reduce the number of doses, relative to immediate-release medications, which may help maintain consistency through scheduled treatments. ${ }^{14,20}$

Paliperidone palmitate (PP) is an atypical antipsychotic medication indicated for schizophrenia. Paliperidone was approved by the Food and Drug Administration (FDA) in 2006 for the treatment of schizophrenia. The long-acting injectable form of paliperidone was approved by the FDA on July 31, 2009 for the treatment of schizophrenia, and subsequently approved in Europe in 2011. The long-acting form of paliperidone is injected into the patient by a health care professional on a monthly basis, which may help with adherence rates. The medication has been shown, in both short-term and long-term clinical trials, to delay relapse time and help provide symptom control for patients experiencing acute exacerbation of schizophrenia. ${ }^{21,22}$ As a result, the consistent use of long-acting, injectable PP may reduce relapse rates and hospitalization, potentially leading to a reduction in the economic burden associated with schizophrenia.

The goal of this study is to estimate the reduction in hospitalization costs associated with the use of long-acting, injectable PP in the People's Republic of China, Korea, and Malaysia relative to alternative treatment medications. In the analysis, we combined localized cost data for the three individual countries with data on the reduction in hospitalization rates derived from a recent Phase IIIB trial (clinical registry number CR016522). ${ }^{23}$ Results suggest that reductions in hospital utilization cost may occur through the use of long-acting PP relative to alternatives.

\section{Methodology and data}

The study focuses exclusively on the estimated reduction in hospitalization days following treatment with PP and the associated cost savings. Cost analysis was done using a 
payer's perspective and only includes the direct health care costs associated with hospitalization. All monetary values were converted into US dollars to help facilitate comparisons with existing studies. The analysis was done over two time horizons: 12 months and 18 months after therapy begins. In both cases, mean per patient year hospitalization costs were compared to a retrospective period of 12 months prior to the beginning of therapy with PP. Subset analysis was performed on patients with $<1$ year of schizophrenia history versus those between 1 year and 5 years of schizophrenia history. Since we annualized the duration of hospital stays, this may exaggerate the length of treatment duration for those with $<1$ year of history.

Localized, direct medical care costs per hospital day for the People's Republic of China were taken from a literature review on the burden of schizophrenia in the People's Republic of China. ${ }^{24}$ The review cites a 2010 study that examined the direct psychiatric hospital costs for 3,117 patients diagnosed with schizophrenia. The median total cost for patients with schizophrenia was found to be $¥ 10,765$ (US\$1,722) per admission or $\sim 241$ (US\$38.56) per day. The inpatient cost per day for Korea was taken from a direct medical cost analysis in hospitalized patients with schizophrenia set in Korea. ${ }^{25}$ The study used $¥ 129,171$ (US\$118.84) as the cost per inpatient hospitalization day in 2010. Hospitalization costs per day for Malaysia were based on estimates from the WHO. The estimated cost per bed day for primary hospitals in Malaysia for 2005 was US\$68.46. ${ }^{26}$

In order to obtain the heath care resource utilization for patients diagnosed with schizophrenia, we used outcomes from a recent Phase IIIB study. ${ }^{23}$ The background information of the study, such as patients treatment status at baseline and recruitment methodology has been published previously. ${ }^{23}$ The primary objective of the study was to explore safety, tolerability, and treatment response of PP once-monthly injections compared to different previous antispsychotic medications in patients with schizophrenia. Enrollment in the study was limited to recently diagnosed patients with schizophrenia, defined in this case as having received the diagnosis within a 5-year period prior to screening. The health care utilization analysis set included 108 total subjects from the People's Republic of China, 109 total subjects from Malaysia, and 94 total subjects from Korea. Hospital utilization rates were calculated for the 12-month period prior to beginning treatment with PP and for both 12 months and 18 months following the beginning of treatment. Focus was placed on full institutionalized days per year where full institutionalization was defined as a continuous 12-24-hour period of hospitalization that included at least one overnight stay. All the results from the study were converted to institutionalized days per person year. The mean $(95 \% \mathrm{CI})$ numbers of fully institutionalized days per person year in the People's Republic of China were found to be $63.26(41.16,85.36)$ during the 12-month retrospective period, $17.49(3.05,31.94)$ days per person year during the initial 12 months of the prospective period, and $17.09(2.68,31.50)$ days per person year during the full 18-month prospective period. Additionally, when individuals with a diagnosed history of schizophrenia of $<1$ year at the time of enrollment were excluded, the mean numbers of fully institutionalized days per person year were found to be $50.70(24.52,76.87)$ during the 12-month retrospective period, $15.21(0.00,32.18)$ days per person year during the initial 12-month prospective period, and 14.82 $(0.00,31.72)$ days per person year during the full 18 -month prospective period.

In Korea, the mean numbers of fully institutionalized days per person year were found to be $55.68(32.09,79.27)$ during the 12-month retrospective period, $9.41(0.00,20.37)$ days per person year during the 12-month prospective period, and $8.38(0.00,19.18)$ days per person year during the full 18-month prospective period. Excluding individuals with a diagnosed history of schizophrenia of $<1$ year at the time of enrollment, the mean numbers of fully institutionalized days per person year were found to be $43.99(14.23,73.76)$ during the 12-month retrospective period, $13.15(0.00,30.36)$ days per person year during the 12-month prospective period, and $12.02(0.00,29.04)$ days per person year during the full 18-month prospective period.

The mean numbers of fully institutionalized days per person year in Malaysia were found to be 131.40 (101.26, 161.53 ) during the 12-month retrospective period, 42.27 $(22.86,61.68)$ days per person year during the 12-month prospective period, and $40.15(21.33,58.96)$ days per person year during the full 18-month prospective period. Excluding individuals with a diagnosed history of schizophrenia of $<1$ year at the time of enrollment, the mean numbers of fully institutionalized days per person year were found to be $145.51(104.83,186.18)$ during the 12-month retrospective period, $49.92(22.90,76.94)$ days per person year during the 12-month prospective period, and $46.64(20.74,72.53)$ days per person year during the full 18-month prospective period. In all the cases, the reduction in full institutionalized days following the initiation of treatment with PP relative to the retrospective period were found to be statistically significant $(P<0.0001)$ using a paired $t$-test. 


\section{Results}

Analysis was done individually for each of the three countries by utilizing the full institutionalized days per person year results from the Phase IIIB study and the localized, direct inpatient health care utilization costs derived from published literature sources (Table 1). Note that the variation in the costs for each country may reflect the differences in purchasing power and per capita gross domestic product across the countries. For People's Republic of China, the estimated total per patient year hospitalization costs during the retrospective period were found to be $\$ 2,439$ for the full sample and $\$ 1,955$ for the sample excluding patients diagnosed within 1 year prior to enrollment in the sample. During the initial 12-month period following treatment with PP, the total per patient year hospitalization costs were $\$ 674$ and $\$ 586$ for the full sample and sample excluding newly diagnosed cases, respectively. After adjusting for different sample sizes in the study periods, this resulted in an estimated cost reduction of $\$ 1,991$ and $\$ 1,567$ per patient year. All of these cost reductions are due to the reduced number of hospital utilization days following treatment. During the entire 18-month period during treatment, the total per patient year costs were found to be $\$ 659$ and $\$ 571$, respectively, for the two samples, resulting in cost reductions of $\$ 2,007$ and $\$ 1,583$ relative to the retrospective period.

In doing the analysis for Korea, results for the retrospective period were $\$ 6,617$ for the full sample and $\$ 5,228$ for the sample excluding patients diagnosed within 1 year prior to enrollment in the sample. During the initial 12-month period following treatment, annual per patient costs were found to be $\$ 1,118$ and $\$ 1,563$ for the full sample and sample excluding newly diagnosed cases, respectively. The resulting per patient year cost reductions were $\$ 6,698$ and $\$ 5,408$ for each sample relative to the retrospective period. When the entire 18-month prospective period is considered, the costs were estimated to be $\$ 996$ and $\$ 1,428$ for each sample, resulting in per patient year cost reductions of $\$ 6,821$ and $\$ 5,543$ relative to the retrospective period.

Analysis for Malaysia resulted in estimated total per patient year hospitalization costs during the retrospective period of $\$ 8,996$ for the full sample and $\$ 9,962$ for the sample excluding patients diagnosed within 1 year prior to enrollment in the sample. During the initial 12-month period following treatment with PP, the total per patient year hospitalization costs were $\$ 2,894$ and $\$ 3,418$ for the full sample and sample excluding newly diagnosed cases, respectively. This resulted in estimated cost reductions of $\$ 6,716$ and $\$ 7,444$ per patient year. During the entire 18-month period

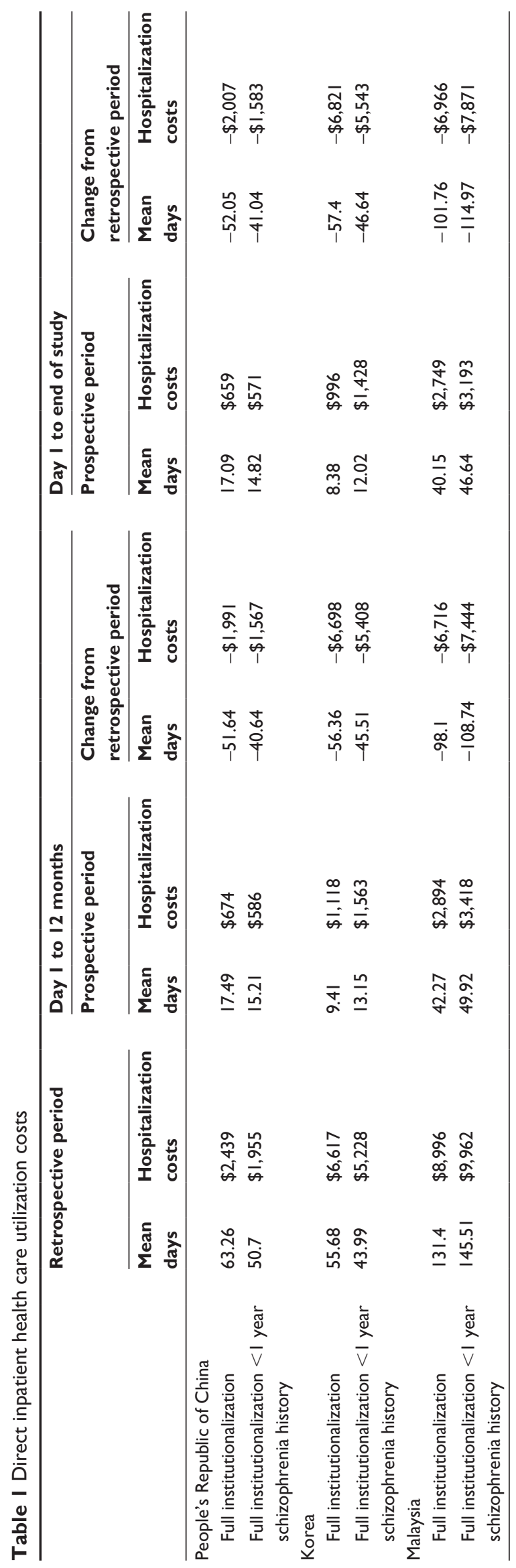


following treatment, the total per patient year costs were found to be $\$ 2,749$ and $\$ 3,193$, respectively, for the two samples, resulting in cost reductions of $\$ 6,966$ and $\$ 7,871$ relative to the retrospective period.

\section{Discussion}

In this paper, we have attempted to further analyze the health care resource utilization costs of patients with schizophrenia in the People's Republic of China, Korea, and Malaysia with specific focus on the reduction in hospitalization costs associated with the use of long-acting, injectable PP relative to alternative treatment medications. In the analysis, we combined localized cost data for the three individual countries with data on the reduction in hospitalization rates derived from a recent Phase IIIB trial. Results suggest that reductions in hospital utilization cost may occur through the use of long-acting PP relative to alternatives.

There have been several studies that have looked at the costs associated with schizophrenia; however, the majority of these studies have been conducted on the US and Europe populations, and there are a limited number of studies addressing this issue for Asia. ${ }^{27}$ To our knowledge, this is the first paper to specifically address the reduction in hospitalization costs for patients in Asia resulting from the treatment using PP relative to alternative medications currently in use for the treatment of schizophrenia.

This study as implemented is a subject to a number of potential limitations, which may impact the overall results. The study was conducted from a third party payer perspective instead of using a societal perspective. As a result, we do not include the additional cost savings, such as the value of lost productivity, physical and social difficulties, and caregiver time costs, associated with treatments more likely to result in adherence. Given the substantial economic burden associated with schizophrenia, both direct and indirect, the inclusion of these costs would likely have added to the potential cost reductions from the use of PP relative to alternative medications. The analysis also required extrapolation of several localized cost parameters from published sources combined with health care utilization data from a clinical study. We note that the process of incorporating data derived from a variety of sources is subject to bias. The hospitalization costs for Malaysia were drawn from WHO data on hospitalization costs per day and are not specific to mental health or schizophrenia. As a result, these estimates are subject to additional bias in our results. We also note that, due to cost estimates being derived from published sources, the study lacks more precise estimates on hospital utilization costs. We also note that the data on hospital utilization rates were derived from a Phase IIIB study using a mirror analysis approach rather than double-blind randomized control methodology. Also, not all data of patients on hospitalization rates within the 12-month retrospective period were available. As such, these patients were not included in the final data analysis. Additionally, in this study, we focused specifically on hospitalization costs. Future studies on determining hospitalization costs specific to schizophrenia are necessary to provide more precise estimates of the cost reduction associated with the use of PP. More advanced modeling techniques, such as budget impact and cost-effectiveness models, depend on such estimates and may not be accurate without these additional studies to inform the model inputs. In that context, we believe that the results of this study will be very useful for future research regarding PP relative to alternative medications for the treatment of schizophrenia in Asia.

\section{Conclusion}

Given the substantial costs associated with the treatment of schizophrenia both worldwide and in Asia, it is important to fully understand costs and outcomes associated with various treatment options. In this study, we have specifically analyzed the direct health care cost savings associated with hospital utilization for patients taking PP relative to alternative treatment methods. The results suggest that per patient year cost reductions could be achieved, likely largely due to increased adherence to treatment.

\section{Acknowledgment}

This paper was directly funded by Janssen-Cilag Asia-Pacific Medical Affairs.

\section{Disclosure}

BCMW, RC, and WF are paid consultants to Janssen. CFC, NK, SL, JW, and FZ are employees of Janssen. The authors report no other conflicts of interest in this work.

\section{References}

1. World Health Organization. Mental Health: Schizophrenia. Available from: http://www.who.int/mental_health/management/schizophrenia/ en/. Accessed October 27, 2014.

2. Centers for Disease Control and Prevention. Burden of Mental Illness. Available from: http://www.cdc.gov/mentalhealth/basics/burden.htm. Accessed October 26, 2014.

3. Andreasen NC, Black DW. Introductory Textbook of Psychiatry. 4th ed. Washington, DC: American Psychiatric Publishing, Inc.; 2006.

4. Mental Health America: Schizophrenia. Available from: http://www. mentalhealthamerica.net/conditions/schizophrenia. Accessed June 30, 2015.

5. Li N, Chen G, Du W, Song X, Zhang L, Zheng X. Population-level prevalence estimate and characteristics of psychiatric disability among Chinese adults. J Psychiatr Res. 2011;45(11):1530-1534. 
6. Zhai J, Guo X, Chen M, Zhao J, Su Z. An investigation of economics costs of schizophrenia in two areas of China. Int J Ment Health Syst. 2013; 7(1):26.

7. Chang SM, Cho SJ, Jeon HJ, et al. Economic burden of schizophrenia in South Korea. J Korean Med Sci. 2008;23(2):167-175.

8. Park SC, Lee MS, Kang SG, Lee SH. Patterns of antipsychotic prescription to patients with schizophrenia in Korea: results from the health insurance review and assessment service-national patient sample. J Korean Med Sci. 2014;29(5):719-728.

9. Yoon CK, Aziz SA. A review of schizophrenia research in Malaysia. Med J Malaysia. 2014;69(suppl A):46-54.

10. Charrier N, Chevreul K, Durand-Zaleski I. The cost of schizophrenia: a literature review. Encephale. 2013;39(suppl 1):S49-S56.

11. Knapp M. Costs of schizophrenia. Br J Psychiatry. 1997;171(Dec): 509-518.

12. Fitch K, Iwasaki K, Villa K. Resource utilization in a commercially insured population with schizophrenia. Am Health Drug Benefits. 2014;7(1):18-26.

13. Goeree R, Farahati F, Burke N, et al. The economic burden of schizophrenia in Canada in 2004. Curr Med Res Opin. 2005;21:2017-2028.

14. Carter NJ. Extended-release intramuscular paliperidone palmitate: a review of its use in the treatment of schizophrenia. Drugs. 2012;72(8): $1137-1160$.

15. National Institute for Health and Clinical Excellence. Schizophrenia: core interventions in the treatment and management of schizophrenia in adults in primary and secondary care (updated edition). National clinical guideline number 82 [online]. Available from (http://www. nice.org.uk/guidance/cg82). Accessed April 2, 2015.

16. Lehman AF, Lieberman JA, Dixon LB, et al. Practice guideline for the treatment of patients with schizophrenia, second edition. Am J Psychiatry. 2004;161(2 suppl):1-56.

17. Robinson D, Woerner MG, Alvir JM, et al. Predictors of relapse following response from a first episode of schizophrenia or schizoaffective disorder. Arch Gen Psychiatry. 1999;56(3):241-247.
18. The Scottish Schizophrenia Research Group. The Scottish first episode schizophrenia study VIII. Five-year follow-up: clinical and psychosocial findings. Br J Psychiatry. 1992;161(4):496-500.

19. Gilday E, Nasrallah HA. Clinical pharmacology of paliperidone palmitate a parenteral long-acting formulation for the treatment of schizophrenia. Rev Recent Clin Trials. 2012;7(1):2-9.

20. Nasrallah HA. The case for long-acting antipsychotic agents in the post-CATIE era. Acta Psychiatr Scand. 2007;115:260-267.

21. Hough D, Gopal S, Vijapurkar U, Lim P, Morozova M, Eerdekens M. Paliperidone palmitate maintenance treatment in delaying the time-torelapse in patients with schizophrenia: a randomized, double-blind, placebo-controlled study. Schizophr Res. 2010;116(23):107-117.

22. Gopal S, Hough DW, Xu H, et al. Efficacy and safety of paliperidone palmitate in adult patients with acutely symptomatic schizophrenia: a randomized, double-blind, placebo-controlled, dose-response study. Int Clin Psychopharm. 2010;25(5):247-256.

23. Zhang F, Si T, Chiou CF, et al. Efficacy, safety, and impact on hospitalizations of paliperidone palmitate in recent-onset schizophrenia. Neuropsychiatr Dis Treat. 2015;11:657-668. [eCollection 2015].

24. Montgomery W, Liu L, Stensland MD, Xue HB, Treuer T, AscherSvanum H. The personal, societal, and economic burden of schizophrenia in the People's Republic of China: implications for antipsychotic therapy. Clinicoecon Outcomes Res. 2013;5:407-418.

25. Kim B, Lee TJ, Woo JM, Park JI, Kwon JS. Cost-utility analysis of paliperidone palmitate long acting injection (PLAI) versus oral atypical antipsychotics in non-adherent schizophrenia patients in South Korea. Korean J Psychopharmacol. 2012;23:17-27. [Korean].

26. World Health Organization. Available from: http://www.who.int/ choice/country/mys/cost/en/. Accessed October 27, 2014.

27. Millier A, Shupo F, Chauhan D, Toumi M. Economic burden in schizophrenia: a literature review. Value Health. 2012;15(7):A336.
Neuropsychiatric Disease and Treatment

\section{Publish your work in this journal}

Neuropsychiatric Disease and Treatment is an international, peerreviewed journal of clinical therapeutics and pharmacology focusing on concise rapid reporting of clinical or pre-clinical studies on a range of neuropsychiatric and neurological disorders. This journal is indexed on PubMed Central, the 'PsycINFO' database and CAS,

\section{Dovepress}

and is the official journal of The International Neuropsychiatric Association (INA). The manuscript management system is completely online and includes a very quick and fair peer-review system, which is all easy to use. Visit http://www.dovepress.com/testimonials.php to read real quotes from published authors. 